STRUCTURAL SCIENCE CRYSTAL ENGINEERING MATERIALS

ISSN 2052-5206

\section{Multi-Component Crystals. Synthesis, Concepts, Function. Edited by Edward Tiekink and Julio Zukerman- Schpector. De Gruyter, 2017, Hardcover, Pp. xv+347. Price EUR 139.95, USD 160.99, GBP 127.88. ISBN 978-3- 11-046365-1}

\author{
Enrique Espinosa*
}

Université de Lorraine, CNRS, CRM2, Nancy, France. *Correspondence e-mail: enrique.espinosa@univ-lorraine.fr

Multi-component crystals (or redundantly, multi-component co-crystals, as the term is sometimes found in the literature, in spite of the fact that any co-crystal is, by definition, built from several components) is actually a broad area of research. Modification of the physical and chemical properties of the solid phase of a main component by co-crystallization with one or several co-formers is exploited in an increasing number of applications. In this context, those concerning the pharmaceutical industry are (by number, efforts and invested financial resources) the most significant. Less exploited (but no less important) are applications in other fields of research, such in materials science and supramolecular chemistry. It seems quite natural from the synergetic development of fields that, in the near future, advantage will be taken of the advances achieved in crystal engineering, a relatively young field of research where the area of multi-component crystals develops. Hence, getting insights into the relationship between the electronic properties of molecular systems and their organization in space is expected to lead to the deep understanding of molecular recognition and molecular assembling. These are the quantum crystallographic bases of crystal engineering and, therefore, of the future applications of multi-component crystals, going beyond trial-and-error procedures for synthesizing crystals with tailored properties.

Under the title of Multi-Component Crystals, the book edited by Tiekink and Zukerman-Schpector aims at providing an overview of some of the theoretical and practical aspects, ranging from fundamentals to applications. It spans over 347 pages and is divided in 14 chapters. Chapters 1 and 2 are devoted to the principles of co-crystal formation (mainly in relation to the pharmaceutical industry) and how this approach can be exploited to overcome the problem of poor solubility of drugs, respectively. Chapter 3 introduces electronic descriptors and tools derived from charge density analysis that, pushing crystal engineering towards a quantum crystallography approach, permit the analysis to go well beyond that based on standard determination of molecular structure and molecular packing. Chapter 4 is dedicated to the use of multi-component crystals to tune photochromism of the $N$-salicylideneaniline chromophore by varying its molecular conformation under the effect of the intermolecular environment, which changes with coformers. Chapter 5 concerns the self-assembling of quinoline-based compounds to guide the connection of multiple components. Their use in multi-component syntheses is suitable, as they drive their formation through different kinds of interactions (involving competitive synthons) and are effective in the selection of partners. Chapter 6 deals with the formation of multi-component crystals involving $N$-oxides, which provide a useful range of architectures spanning bifurcated hydrogen-bonding interactions, halogenbonding interactions and bridges between metal ions (to form coordination polymers or metal-organic frameworks). Their polar nature makes them very useful for strengthening the interactions they are involved in and, therefore, for driving molecular assembly. In Chapter 7, several aspects of multi-component crystals, such as synthesis and intermolecular interactions, are discussed upon the application of non-ambient conditions (mainly variable low temperature and/or high pressure). Chapter 8 describes solid-state reactivity and thermal expansion by making use of multi-component crystals. In particular, intermolecular [2+2] photo-dimerization is introduced to discuss how the crystalline proximity of reactive components stimulated by UV light permits the formation of 
molecules that are otherwise extremely difficult to synthesize in solution. Chapter 9 is devoted to a large overview of phase diagrams, along with thermodynamics and kinetics aspects involved in the crystallization of multiple compounds. The impact of these aspects upon co-crystal solubility and dissolution rate is mainly of relevance for pharmaceutical purposes. In Chapter 10, self-assembling patterns involving halogenbonding interactions are discussed in terms of the co-crystal/ salt continuum range and of strong halogen-bonding donors driving main forces in the assembling of molecules. Chapter 11 is devoted to benzene-benzene parallel-stacking interactions of coordinated and non-coordinated benzene molecules in cocrystals, with particular emphasis on horizontal displacements, interaction geometries and energies. Chapter 12 deals with a detailed examination of the Cambridge Structural Database where hydrogen- and halogen-bonding interactions share the same acceptor, namely carbonyl or thiocarbonyl groups. Chapter 13 focuses on the variety and the complexity of cocrystals formed by molecules bearing diamide and pyridyl residues. Here, discussions are complemented by a brief overview of the complexing ability of their neutral, protonated and deprotonated forms towards metal centers. Finally, in the framework of supramolecular chemistry, examples of supramolecular architectures based on main group element lone pair $\cdots \pi$ (arene) interactions (groups III to VI) are discussed in Chapter 14.

In this book, only a few connections are observed between some chapters, and those are not explicitly brought together. The task is left to the savvy reader with enough scientific background. This is regrettable, in particular when dealing with a young and expanding field of research. On the other hand, general aspects of crystal engineering (not necessarily associated with multi-component crystals, or at least not straightforwardly developed for) often appear in the pages as a consequence of the research developed by the contributors. In this respect, the book moves through its chapters with crystal engineering concepts and techniques, whereas in many cases the focus on multi-component crystals appears solely on their consequent applications. Moveover, multi-component crystals with tailored and desired properties are only treated in a few chapters. Accordingly, the reader who is the target of this book will mainly appreciate some of the actual research that takes advantage of well identified patterns (based on specific molecules and functional groups) for driving molecular assemblies with multi-component crystals.

Finally, from a critical reading of this book some more general considerations are suggested. Whereas the scope of the book is not to revisit the broad spectrum of all the aspects of multi-component crystals (too large to make it possible in a single compilation), the absence of real connection between chapters, which are rather independent contributions, makes the book similar to a special issue of a journal, where some articles on a given area of research are published as juxtaposed contributions to the field under a broad title acting as a common denominator. As a consequence, the book is mainly addressed to researchers working in the specific areas treated through the different chapters rather than to students or other readers looking for a general and linked treatment of the subjects. The latter is instead retrieved in 'classical' formats of scientific textbooks, where a single contribution addresses the general approach sought by those readers. Hence, this book resembles in format many others (not only on crystal engineering), gathering scientific advances (mostly reused from early published works) in the same fashion. When scientific work is published in such a way, several questions arise: Where does the relevance of a book stand when compared to special issues of journals (or vice versa)? Is it only a matter of the difference between 'original' articles in special issues and 'mostly reused works' with mini-review-like format in books? If yes, what is the added value with respect to the publication of single articles and short reviews? Moreover, considering the increasing quantity (growing at a rapid rate) of both special issues and book-like publications with a short number of juxtaposed and unconnected contributions, one is led to ask whether they bring any real additional benefit (for readers) more than compiling research outcomes under a more or less related title? Does any additional (scientific) interest remain? What is the goal for publishing houses? It is perhaps time to start reconsidering the way research is published and communicated. 